\title{
Effect of using Femi-band acupressure on primary dysmenorrhea: Randomized controlled trial
}

\author{
Hanan El-Sayed Mohamed *1, Seham Mohamed Salem², ZainabGazar Al-kotb Al-Agamy ${ }^{3}$ \\ ${ }^{1}$ Mansoura University, Mansoura, Egypt \\ ${ }^{2}$ Tanta University, Tanta, Egypt \\ ${ }^{3}$ Fayoum University, Fayoum, Egypt
}

Received: July 29, 2015

DOI: $10.5430 /$ jnep.v5n12p49
Accepted: August 24, $2015 \quad$ Online Published: September 16, 2015

URL: http://dx.doi.org/10.5430/jnep.v5n12p49

\begin{abstract}
Introduction and aim: Dysmenorrhea is one of the most common gynecological disorders. It is affects more than half of menstruating women. The study aim was to investigate the effect of using Femi-band acupressure on primary dysmenorrhea. Methods: Study Design: Randomized controlled trial design was utilized in this study. Study subjects: A purposive sample was used to collect one hundred and fifty students who were randomly divided into two groups an intervention group: consisted of 75 students who applied the acupressure Femi-bands to both legs three times daily for first three days of menstruation with application to each leg for 1 minute. The control group: consisted of 75 students who applied a placebo band three times daily for first three days of menstruation to each leg for 1 minute. Tools: Five tools were be used for data collection: An interviewing schedule, Visual Analogue Scale, Femi-band, Follow up chart and Satisfaction likert scale.

Results: In the intervention group, the severity of pain decreased from 8.27 to 2.92 following intervention, the severity of pain decreased after using the Femi-band (immediately, $30 \mathrm{~min}$, one hour, and 2 hours) at 1st, 2ed, and 3rd month in the intervention group. When the severity of pain was analyzed at each time period, significant differences were observed (immediately, 30 min, one hour, and 2 hours ) after applying the band at 1st, 2ed, and 3rd month.

Conclusions: Intervention group had significant reduction in the severity of dysmenorrhea after using the Femi-band acupressure. Recommendation: Encouraging the use of Femi-band acupressure during the first days of menstruation to reduce menstrual pain.
\end{abstract}

Key Words: Primary dysmenorrhea, Femi-band, Acupressure

\section{INTRODUCTION}

Dysmenorrhea is one of most common gynecological disorders. It affects more than 50 percent of menstruating women. ${ }^{[1]}$ Dysmenorrhea is uterine pain around the time of menses. Pain may occur with menses or precede menses by 1 to 3 days. Pain tends to peak $24 \mathrm{~h}$ after onset of menses and subside after 2 to 3 days. ${ }^{[2]}$ Dysmenorrhea can be classified into two different types: primary and secondary. Primary dysmenorrhea is defined as painful menstruation, in women with normal anatomy of the pelvis, that usually begins in adolescence. The onset of primary dysmenorrhea is usually 6 to 12 months after menarche, which coincides with the occurrence of regular ovulatory cycles. ${ }^{[3]}$ Secondary dysmenorrhea is menstrual pain associated with underlying pathology, and its onset may be years after menarche. ${ }^{[2]}$

The prevalence of primary dysmenorrhea varies between $50 \%$ and $90 \% .{ }^{[4]}$ In an epidemiological study that entailed 664 secondary school students from urban and rural areas

*Correspondence: Hanan El-Sayed Mohamed; Email: alemam2100@yahoo.com; Address: Mansoura University, Mansoura, Egypt. 
in Mansoura, Egypt reported that $75 \%$ of the students have dysmenorrhea, rated mild in $55.3 \%$, moderate in $30 \%$, and severe in $14.7 \% .^{[5]}$ Also another study indicated ${ }^{[6]}$ a high prevalence of dysmenorrhea, that is, $78.8 \%$ among technical secondary schools girls in Mansoura, Egypt.

The main cause of dysmenorrhea is an increased production of endometrial prostaglandin, resulting in a high concentration of prostaglandins in blood which causes uterine contractions, ischemia, cramping, and pelvic pain. ${ }^{[7]}$

Dysmenorrhea can reduce productivity, creativity, and work performance due to serious daily stress as well as social and economic loss. Dysmenorrhea typically lasts approximately 48-72 hours and includes symptoms such as lower abdominal cramps, back pain, nausea, vomiting, loss of appetite, fatigue, and nervousness. ${ }^{[8]}$

The treatment approach for primary dysmenorrhea includes pharmacological as well as non-pharmacological therapeutic methods. Pharmacological approaches may not be completely effective because of the side effects experienced by approximately $15 \%$ of women with primary dysmenorrhea. In addition, young Egyptian girls prefer not to use medication for dysmenorrhea as they believe that it may affect fertility or cause medication or drug dependence. ${ }^{[9]}$

A variety of alternative methods have been used for the treatment of dysmenorrhea such as acupuncture, acupressure, Transcutaneous Electronic Nerve Stimulation (TENS), biofeedback, herbal therapy, and complementary medicine. ${ }^{[10]}$

Acupressure is non-pharmacological treatments which are highly regarded nowadays it is the use of touch technique to balance energy channels in the body. ${ }^{[11]}$ Energy or cosmic life force, which is called "Qi" in Chinese, moves inside the body in certain paths or channels, called meridian. Energy flow in these meridians is in balance. If energy is reduced in one or more meridians, body health would be affected. There are some parts in these meridians which have the minimal energy. These are the different points used in traditional medicine to utilize needle or acupressure. ${ }^{[12]}$

Sanyinjiao (SP6) or the meeting point of spleen, liver and kidney channels, is located on spleen meridian, which is four fingers above the inner ankle behind the posterior edge of tibia. ${ }^{[13]}$ This point is considered as a selective point in treating women's diseases. It is easily accessible, can be simply found and pressure can be exerted on it without the help of medical staff. ${ }^{[14]}$ So the purpose of this study was to investigate the effect of using Femi-band acupressure on primary dysmenorrhea.

\subsection{Aim of the study}

The aim of this study was to investigate the effect of using Femi-band acupressure on primary dysmenorrhea.

\subsection{Research hypothesis}

The Femi-band acupressure will alleviate primary dysmenorrhea among students of Technical Institute of Nursing students, Tanta University, Egypt.

\section{SUBJECTS AND METHOD}

\subsection{Study design}

A randomized controlled trial design was utilized in this study.

\subsection{Study setting}

This study was conducted at Technical Institute of Nursing, Tanta University, Egypt. From the period of October 2013 to June 2014.

\subsection{Subjects of the study}

A purposive sample was used to collect one hundred and sixty female nursing students who complain of primary dysmenorrhea. Ten students were excluded from the study, seven of them were taking analgesics during their menses and the other three were unable to follow up with the study regimen. One hundred and fifty students were randomly divided into two groups: Group (A): Intervention group: consisted of 75 students who applied the acupressure Femi-band to both legs three times daily for the first three days of menstruation with an application1 minute to each leg on SP6 point, which located four fingers widths above the inner ankle bone posterior border of the tibia. Group (B): Control group: consisted of 75 students who applied the placebo band to both legs three times daily for the first three days of menstruation, each one for 1 minute, which located six fingers widths above the inner ankle bone posterior border of the tibia.

\subsection{Inclusion criteria}

- Aged 18-22 year-old

- Suffer from moderate to severe primary dysmenorrhea (score of three or more on a Visual Analog Scale)

- Regular cycles 21 to 35 days lasting three to seven days

- Receiving no analgesics during the study period

- No hormonal replacement therapy during the last 6 months

- Have no systemic or gynecologic disease.

\subsection{Tools of data collection}

Five tools were used for data collection. It is consisted of: An interviewing schedule, Visual Analogue Scale, Femi-band 
and follow up chart and satisfaction likert scale.

Tool (1): An interviewing Questionnaire Schedule. It was developed by the researcher after extensive review of relevant and recent literature. It includes two parts: Part one: includes general characteristics such as: age, residence. Part two: includes menstrual history such as age of menarche, duration of menstruation, frequency of menstruation, starting time of dysmenorrhea, ending time of dysmenorrhea, limited daily activity.

Tool (2): A Visual Analogue Scale (VAS). It was adopted from Crichton ${ }^{[15]}$ and used by the researcher to assess the severity of pain before and after intervention. It is a selfreported $10 \mathrm{~cm}$ straight line which represents the pain intensity, the two opposite ends representing no pain to pain as bad as it could be in between these two phrases, words like: Slight pain, mild pain, moderate pain, severe pain, and very severe pain are assigned to each $2 \mathrm{~cm}$ distance respectively.

Tool (3): Femi-band (Bracelet for treating pain of menstrual cycle): It was designed by researcher (Salem S,M., 1195/2013 Egyptian Patent Office). ${ }^{[16]}$ Femi-band is a band used to relieve dysmenorrhea by applying pressure and massage in the same time on SP6 point, which located four finger widths above the inner ankle bone posterior border of the tibia. The band has a button of half to one $\mathrm{cm}$, height for pressure, and vibration generat or behind the button connected with battery and on/off key. The pressure is applied and controlled by changing the size of the band according to the size of the girl's leg at the site of SP6 point (for intervention group). A band without button and vibration generator to be placebo band for (for control group).

Tool (4): Follow up chart: It is a daily chart developed by the researcher after extensive review of relevant and recent literature. It recorded by the subjects to assess the severity of dysmenorrhea for three months (before using the band, Immediately, $30 \mathrm{~min}, 1$ and 2 hours after intervention).

Tool (5): Satisfaction likert scale: It was used to assess satisfaction of subjects after using Femi-band and placebo band. It was scored as the following: unsatisfied (1), satisfied (2).

A 3 jury from experts in the obstetric nursing field tested the content validity. According to expert's suggestions the tool was modified.

\subsection{A pilot study}

A pilot study was conducted on 15 (10\%) subjects from the mentioned setting to measure the feasibility of the study setting, content validity of the tools and time required for the completion of each tool. Results obtained were useful in appraisal and modification of the tools; these subjects were

Published by Sciedu Press later excluded from the study sample.

\subsection{Field work}

- Collection of data covered a period of 9 months from October 2013 to June 2014. All subjects signed a written consent followed by a baseline interview. During the interview, the collected data included student's age, residence, age of menarche, length of menstrual cycle, duration of menstrual flow, level and duration of pain, starting and ending of pain, hen the intervention and control groups were selected randomly where the odd numbers were recruited as intervention group and the even numbers are recruited as control group.

- Intervention group: The researcher teach the subjects how to position the Femi-band correctly as follow: The pressure is applied and controlled by changing the size of the band according to the size of the girl's leg at the site of SP6 point, which located four finger widths above the inner ankle bone posterior border of the tibia.

- The researcher teach the subjects to determine the SP6 point, then suit the size of the band according to the size of the subject' leg (tide the band to apply pressure by the button in depth half to one $\mathrm{cm}$ ), then press the on/off key to let the vibration generat or do massage during the pressure. This process to be applied for one minute, three times daily started with menstrual flow for three days for three months.

- Control group: The researchers give them band without button and vibration generator and teach them to apply it at six fingers widths above the inner ankle bone posterior border of the tibia, for one minute three times daily for 3 days from the beginning of menstrual flow.

- Both groups were instruct to measure pain intensity before applying band then immediately, $30 \mathrm{~min}$, one hour, and two hour following the intervention for three consecutive months.

\subsection{Statistical analysis}

Statistical Package for Social Sciences (SPSS) version 21 was used for quantitative data analysis. Quality control was done at the stages of coding and data entry. Data were presented using descriptive statistics in the form of frequencies and percentages for qualitative variables, and means and standard deviations for quantitative variables. Qualitative variables were compared using $T$ test and Correlation $(r)$ test. Statistical significance was considered at $p$-value $<$ .05 , highly significant difference obtained at $P<.01$ and non-significant difference obtained at $P>.05$. 


\subsection{Ethical considerations}

Written consent was obtained from the students included in the sample. They were reassured about the confidentiality of the information. They were informed about their rights to refuse participation or withdraw at any time. The study maneuvers couldn't entail any harm to participants.

\section{RESUltS}

Table 1 showed that there were no statistically significant differences among the two groups with respect to age, residence, the menarche age, duration, cycle and amount of menstruation, first time of dysmenorrhea, starting and ending time of dysmenorrhea and limited daily activity $(p>.05)$.

Table 1. Frequency distribution of the study subjects according to general and menstrual characteristics

\begin{tabular}{|c|c|c|c|c|c|}
\hline \multirow{2}{*}{ Variables } & \multicolumn{2}{|c|}{ Intervention } & \multicolumn{2}{|c|}{ Control } & \multirow{2}{*}{$p$ value } \\
\hline & $\mathbf{N}$ & $\%$ & $\mathbf{N}$ & $\%$ & \\
\hline \multicolumn{6}{|l|}{ Age (years) } \\
\hline $18-$ & 32 & 42.7 & 22 & 29.3 & \multirow{3}{*}{.295} \\
\hline 19- & 21 & 28.0 & 20 & 26.7 & \\
\hline $20-$ & 22 & 29.3 & 33 & 44.0 & \\
\hline \multirow{3}{*}{$\begin{array}{l}\text { Residence } \\
\text { Urban } \\
\text { Rural }\end{array}$} & & & & & \multirow{3}{*}{.050} \\
\hline & 30 & 40.0 & 33 & 44.0 & \\
\hline & 45 & 60.0 & 42 & 56.0 & \\
\hline \multirow{3}{*}{$\begin{array}{l}\text { Age of menarche (years) } \\
11- \\
13-\end{array}$} & 64 & 85.3 & 45 & 60.0 & \multirow{3}{*}{.327} \\
\hline & 11 & 14.7 & 30 & 40.0 & \\
\hline & & & & 40.0 & \\
\hline \multirow{3}{*}{$\begin{array}{l}\text { Duration of menstruation (day) } \\
3-\end{array}$} & 38 & 50.7 & 35 & & \multirow{3}{*}{.253} \\
\hline & 37 & 49.3 & 40 & 53.3 & \\
\hline & & & & & \\
\hline \multirow{2}{*}{$\begin{array}{l}\text { Cycle of Menstruation } \\
\text { 21- }\end{array}$} & 50 & 66.7 & 33 & 44.0 & \multirow[b]{2}{*}{.514} \\
\hline & 25 & 33.3 & 42 & 56.0 & \\
\hline \multirow{3}{*}{$\begin{array}{l}\text { Amount of menstruation } \\
\text { Medium } \\
\text { Large }\end{array}$} & & & & & \multirow{3}{*}{.191} \\
\hline & 39 & 52.0 & 44 & 58.7 & \\
\hline & 36 & 48.0 & 31 & 41.3 & \\
\hline \multirow{3}{*}{$\begin{array}{l}\text { 1st time of dysmenorrhea } \\
\text { After } 6 \text { month } \\
\text { After one year }\end{array}$} & 51 & 68.0 & 36 & 48.0 & \multirow{3}{*}{.744} \\
\hline & 24 & 320 & 39 & 520 & \\
\hline & & 32.0 & 39 & & \\
\hline \multirow{3}{*}{$\begin{array}{l}\text { Starting time of dysmenorrhea } \\
\text { Before menstruation } \\
\text { 1st day of menstruation }\end{array}$} & & & & & \multirow{3}{*}{.072} \\
\hline & 72 & $\begin{array}{l}4.0 \\
960\end{array}$ & 19 & 25.3 & \\
\hline & & & & & \\
\hline \multirow{3}{*}{$\begin{array}{l}\text { Ending time of dysmenorrhea } \\
\text { 2nd day } \\
\text { 3rd day }\end{array}$} & & & & & \multirow{3}{*}{.870} \\
\hline & 15 & 80 & 14 & $\begin{array}{l}18.7 \\
813\end{array}$ & \\
\hline & 15 & 20 & 61 & 81.3 & \\
\hline \multirow{4}{*}{$\begin{array}{l}\text { Limited daily activity } \\
\text { Slightly } \\
\text { Medially affected } \\
\text { Seriously affected }\end{array}$} & 30 & 40.0 & 30 & 40.0 & \multirow{4}{*}{.109} \\
\hline & 20 & 26.7 & 31 & 41.3 & \\
\hline & 25 & 33.3 & 14 & 18.7 & \\
\hline & & & & & \\
\hline
\end{tabular}

Table 2 presented the using band according to instruction and frequency of using band among study groups. Most of the study subjects $94.7 \%$ and $93.3 \%$ for intervention and control group respectively using band according to instruction. More than half of subjects $(56.0 \%)$ of intervention group using band three times per day compare to $70.7 \%$ of control group. There were highly statistical significant difference between intervention and control group $(p=.001)$.

Figure 1 presented the satisfaction after using band among study groups. All the study subjects $(100 \%)$ in the intervention group were satisfied; diverse they weren't satisfied in the majority of the control group (85.3\%). There were highly statistical significant difference between intervention and control group $(p=.000)$.

Table 3 showed the comparisons of the severity of pain between intervention and control groups during three months. Dysmenorrheal score at various intervals before using the band and after using the band (immediately, $30 \mathrm{~min}$, one hour, and 2 hours). In the intervention group, the severity of pain decreased from 8.27 to 2.92 following intervention, while severity ratings also decreased in the control group from 8.83 to 7.85 at 1 st month. The table showed the severity 
of pain decreased after using the Femi-band (immediately, 30 observed (immediately, 30 min, one hour, and 2 hours) after min, one hour, and 2 hours) at 1st, 2ed, and 3rd month in the applying the band at 1st, 2ed, and 3rd month. No difference intervention group. Statistical significant differences were was observed at before using the band at 1st month.

Table 2. Frequency distribution of the study subjects according to use of band

\begin{tabular}{|c|c|c|c|c|c|}
\hline \multirow{2}{*}{ Items } & \multicolumn{2}{|c|}{ Intervention } & \multicolumn{2}{|c|}{ Control } & \multirow{2}{*}{$p$ value } \\
\hline & $\mathbf{N}$ & $\%$ & $\mathbf{N}$ & $\%$ & \\
\hline \multicolumn{6}{|c|}{ Using band according to instruction } \\
\hline Yes & 71 & 94.7 & 70 & 93.3 & \multirow[t]{2}{*}{.731} \\
\hline No & 4 & 5.3 & 5 & 6.7 & \\
\hline \multicolumn{5}{|l|}{ Frequency of using band } & \multirow{6}{*}{.001} \\
\hline Once & 4 & 5.3 & 0 & 0 & \\
\hline Twice & 11 & 14.7 & 0 & 0 & \\
\hline Three times & 42 & 56.0 & 53 & 70.7 & \\
\hline More than three times & 2 & 2.7 & 0 & 0 & \\
\hline In needed & 16 & 21.3 & 22 & 29.3 & \\
\hline
\end{tabular}

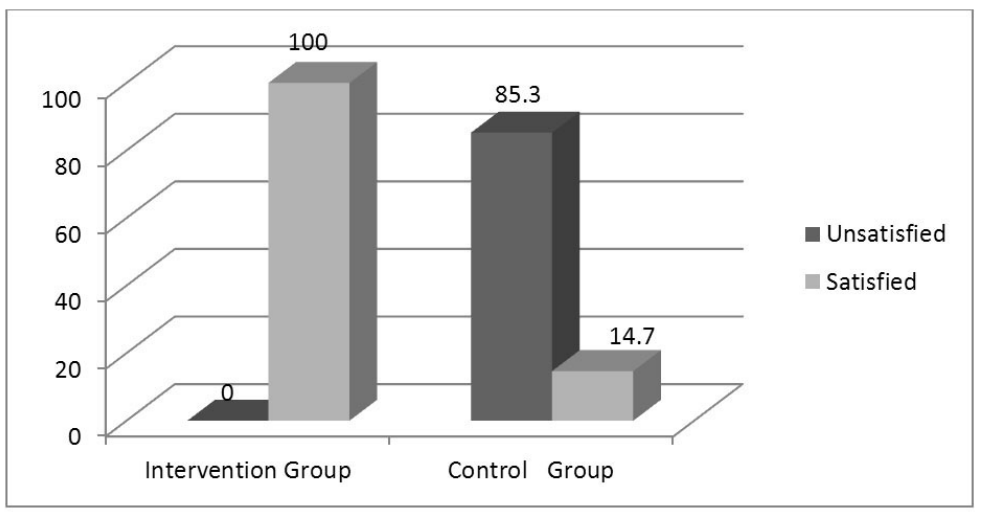

Figure 1. Frequency distribution of the study subjects according to satisfaction after using band

Table 3. Comparison of the severity of pain between intervention and control groups during three months

\begin{tabular}{llll}
\hline \multirow{2}{*}{ Pain score at various intervals } & Intervention group & Control group & p-value \\
\cline { 2 - 3 } & Mean \pm SD & Mean \pm SD & .102 \\
\hline During 1st month & & & .003 \\
Before using the band & $8.27 \pm 1.044$ & $8.83 \pm 1.369$ &, 000 \\
Immediately after using & $6.87 \pm 1.256$ & $8.27 \pm 1.178$ & .000 \\
After half an hour & $5.12 \pm 1.039$ & $7.68 \pm 1.499$ & .000 \\
After an hour & $2.92 \pm 1.205$ & $8.00 \pm 1.542$ & .000 \\
After 2 hours & $1.19 \pm 0.911$ & $7.85 \pm 0.849$ & .000 \\
During 2nd month & & & .000 \\
Before using the band & $7.17 \pm 0.828$ & $8.39 \pm 1.173$ & .000 \\
Immediately after using & $5.51 \pm 1.005$ & $7.83 \pm 1.369$ & .000 \\
After half an hour & $4.00 \pm 0.697$ & $7.97 \pm 0.753$ & \\
After an hour & $2.01 \pm 1.020$ & $7.71 \pm 1.303$ & .000 \\
After 2 hours & $0.67 \pm 0.684$ & $7.71 \pm 0.712$ & .247 \\
During 3rd month & & & .000 \\
Before using the band & $5.77 \pm 0.709$ & $8.39 \pm 1.404$ & .000 \\
Immediately after using & $4.53 \pm 0.723$ & $7.83 \pm 1.369$ & .000 \\
After half an hour & $1.96 \pm 1.168$ & $7.83 \pm 1.132$ & $7.85 \pm 1.486$ \\
After an hour & $0.17 \pm 0.381$ & $7.71 \pm 0.897$ & \\
After 2 hours & $0.27 \pm 0.577$ & & \\
\hline
\end{tabular}


Figure 2 showed the severity mean of pain decreased after using the Femi-band (immediately, 30 min, one hour, and 2 hours) at 1st, 2nd and 3rd month in the intervention group.
Statistical significant differences were observed (immediately, $30 \mathrm{~min}$, one hour, and 2 hours) after applying the band at 1st, 2ed, and 3rd month.

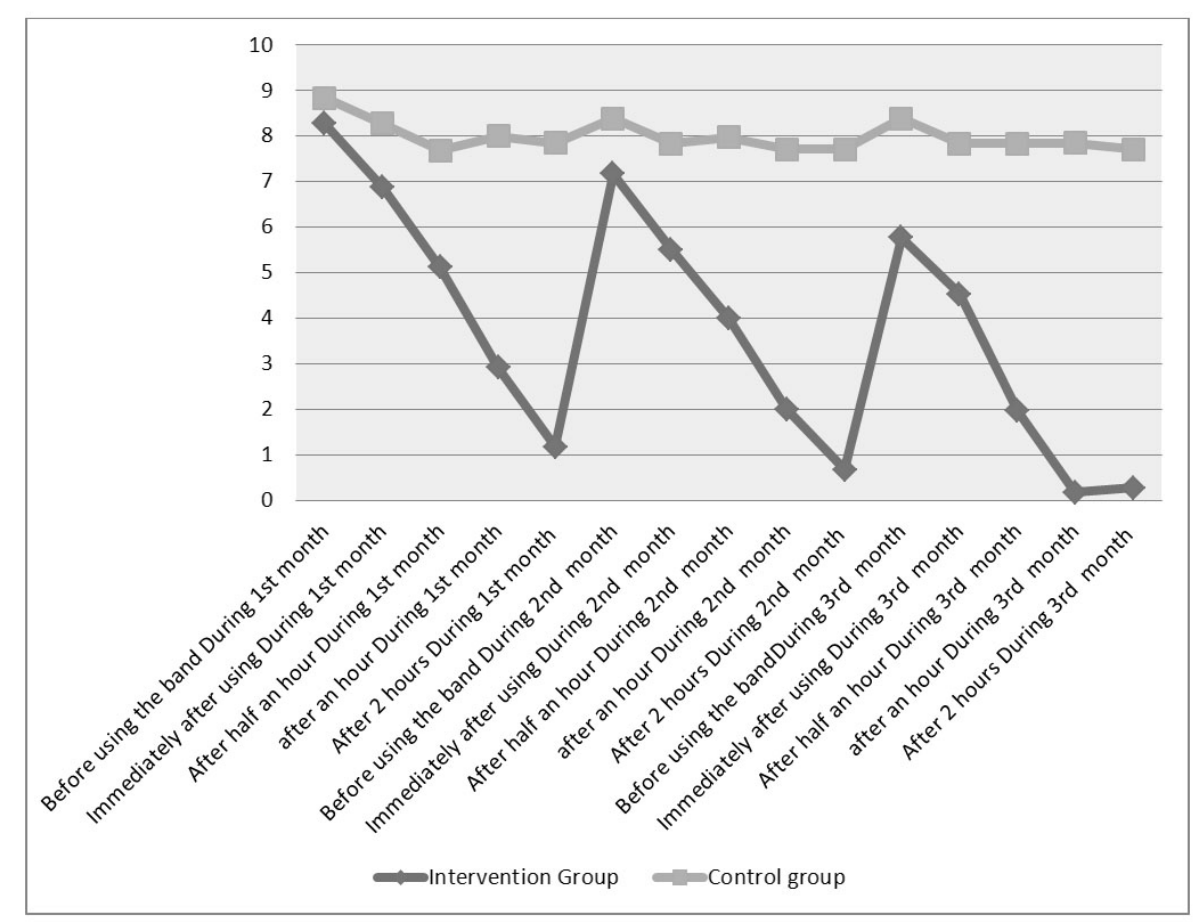

Figure 2. Comparison of the mean severity of pain between intervention and control groups during the three months

\section{Discussion}

The present study was aimed to investigate the effect of using Femi-band acupressure on primary dysmenorrhea among institutional nursing students. This aim was significantly achieved through the present study findings because there was highly significant effect on reduction of the severity of dysmenorrhea intervention group compare to control group. So, hypothesis was accepted i.e., the Femi-band acupressure will alleviate the primary dysmenorrhea.

Femi-band acupressure for dysmenorrhea is a natural way to relieve pain. It is safe, noninvasive, economical and cost free pain relieving technique. Femi-band acupressure can relieve dysmenorrhea. The present study findings showed the severity of pain decreased after using the Femi-band (immediately, $30 \mathrm{~min}$, one hour, and 2 hours) at 1st, 2ed, and 3rd month in the intervention group. When the severity of pain was analyzed at each time period, significant differences were observed (immediately, $30 \mathrm{~min}$, one hour, and 2 hours) after applying the band at 1st, 2ed, and 3rd month. No difference was observed at before using the band at 1 st month.

The present study findings were in agreement with by Chen and Chen ${ }^{[17]}$ who study the effects of acupressure on menstrual distress in adolescent girls: a comparison between
Hegu-Sanyinjiao matched points and Hegu, Zusanli single point and reported that acupressure was effective in reducing severity of dysmenorrhea when it was given for 20 minutes on SP6 point. Also the study results in the same line with Wong et al. ${ }^{[18]}$ who determined the effectiveness of acupressure by conducting randomized control trial in which acupressure at SP6 was given twice a day for first three days of the menstrual cycle and findings showed statistically significant decrease in pain score.

Furthermore, clinical trial conducted by Gharloghi et al. ${ }^{[19]}$ comparing the acupressure at SP6 and SP8 point in which results showed that the severity of dysmenorrhea diminishes significantly for up to 2 hours following intervention. Moreover the present study findings were in agreement with Sharma et al. ${ }^{[8]}$ who study the effectiveness of acupressure at SP6 point on dysmenorrhea among B.Sc Nursing students and showed that statistically significant reduction in severity of dysmenorrhea. Also the present study findings were in accordance to the study conducted by Chang et al. ${ }^{[20]}$ in which study subjects received acupressure treatment within the first 8 hours of menstruation and showed significant reduction in severity of dysmenorrhea.

Moreover, the current study results were supported by 
Mirbagher et al. ${ }^{[21]}$ who conducted study comparing acupressure at two different points i.e., light touch at SP6 for 20 min in control group and acupressure at SP6 in experimental group and reported that there were significant differences in VAS scores between the experimental and controlgroups immediately, 1, 2, and $3 \mathrm{~h}$ after intervention. Also Study findings were agree with Gharloghi et al. ${ }^{[18]}$ who study the effects of acupressure on severity of primary dysmenorrhea and showed diminished significantly immediately, 30 minutes, 1 hour, and 2 hours after application of acupressure at the SP6 and SP8 points. Furthermore, Kashefi et al. ${ }^{[22]}$ who study the effect of acupressure at the Sanyinjiao point on primary dysmenorrhea and concluded that acupressure at Sanyinjiao point can be an effective, feasible, cost effective intervention for reducing pain of primary dysmenorrhea.

The present study findings showed that all the study subjects in the intervention group were satisfied by using band; diverse they weren't satisfied in the majority of the control group. These findings were supported by Unsal et al. ${ }^{[22]}$ who comparing the effectiveness of acupressure and fish oil capsules and Ibuprofen on pain severity of primary dysmenorrhea and showed that $85 \%$ of participants in acupressure group were satisfied from this method for relieving their dysmenorrhea.

The Femi-band acupressure is a non-pharmacological, costeffective, simple and effective method and more importantly for reducing dysmenorrhea.

\section{Conclusion}

Based on the findings of present study, it was concluded that the intervention group had significant reduction in the severity of primary dysmenorrhea after using the Femi-band acupressure.

\section{RECOMMENDATIONS}

- Encouraging the use of Femi-band acupressure during the first days of menstruation to reduce the menstrual pain.

- Further studies are needed to investigate females acceptance and satisfaction with using the Femi-band acupressure.

- The findings of the study can be used as a guideline for further research on effect of Femi-band acupressure using combination of different points.

- Further investigations are necessary to replicate the beneficial findings of Femi-band acupressure in a large population.

- Further studies are needed to investigate the effect of Femi-band acupressure on another acupressure points as: ST36-TW5-B54-P6-B57.

\section{Conflicts of InTEREST Disclosure}

The authors declare that there is no conflict of interest.

\section{REFERENCES}

[1] Arif Zaidi SM, Khatoon K, Aslam KM. Role of herbal medicine in Ussuruttams (Dysmenorrhoea)J. Acad. Indus. Res. 2012; 1(3): 113-117.

[2] Unsal A, Ayranci U, Tozun M, et al. Prevalence of dysmenorrhea and its effect on quality of life among a group of female university students. Journal of medical Science. 2010; 115(2): 138-145. http://dx.doi.org/10.3109/03009730903457218

[3] Abbaspour Z, Rostami M, Najjar SH. The Effect of Exercise on Primary Dysmenorrhea. Journal of research health science. 2006; 6(1): 26-31.

[4] Sharma P, Malhotra C, Taneja DK, et al. Problems related to menstruation amongst adolescent girls. Indian Journal of Pediatrics. 2008; 75(2): 125-129. http://dx.doi.org/10.1007/s12098-008-0 018-5

[5] El-GilanyA H, Badawi K, El-Fedawy S. Epidemiology of dysmenorrhoea among adolescent students in Mansoura. Eastern Mediterranean Health Journal. 2005; (11): 155-163.

[6] Mohamed H, Neaem S. The Effect of Dysmenorrhea on Quality of Life of Technical Secondary Schools Girls. Medical Journal of Cairo University. 2013; 81(2): 83-89.

[7] Jaafarpour M, Hatefi M, Khani A, et al. Comparative Effect of Cinnamon and Ibuprofen for Treatment of Primary Dysmenorrhea: A
Randomized Double Blind Clinical Trial. Journal of Clinical and Diagnostic Research. 2015; 9(4): 4-7. http://dx.doi.org/10. $7860 / j c d r / 2015 / 12084.5783$

[8] Sharma E, RanaA K, Singh A. An interventional study to assess the effectiveness of acupressure at SP6 point on dysmenorrhea among B.Sc Nursing students. Nursing and Midwifery Research Journal. 2014; 10(4): 145-156.

[9] Rizk SA. Effect of Aromatherapy Abdominal Massage using Peppermint Versus Ginger oils on Primary Dysmenorrhea among Adolescent Girls. Journal of American Science. 2013; 9(11): 597- 605.

[10] Proctor ML, Murphy PA, Pattison HM. Behavioural interventions for primary and secondary dysmenorrhoea. Cochrane Database of Systematic Reviews. 2007; 3.

[11] Bostani KZ, Abadin ZM, Safari A. Compared the effects of acupressure and vitamin $\mathrm{E}$ in the primary dysmenorrheal Saninjiao. Armaghane-danesh. 2009; 14(3): 36-43.

[12] Mohammad S, CharandabiA, Nashtaei MS, et al. The effect of acupressure at the Sanyinjiao point (SP6) on primary dysmenorrhea in students resident in dormitories of Tabriz . Iranian journal of nursing and midwifery research. 2011; 16(4): 1-9.

[13] BeygamA Z, Vijeh M, Latif NR, et al. Effect of acupressure on pain of primary dysmenorrhea. Hayat. 2005; 11(3): 19-28. 
[14] Chen HM, Chen CH. Effects of acupressure at the Sanyinjiao point on primary dysmenorrhoea. J AdvNurs. 2004; 48(4): 380-7. http://dx.doi.org/10.1111/j.1365-2648.2004.03236.x

[15] Crichton N. Visual analogue scale. Journal of Clinical Nursing. 2001; (10): 697-706.

[16] Salem SM. Bracelet for treating pain of menstrual cycle. Egyptian Patent Office. 2013; 1195.

[17] Chen HM, Chen CH. Effects of acupressure on menstrual distress in adolescent girls: a comparison between Hegu- Sanyinjiao matched points andHegu, Zusanli single point. Journal of Clinical Nursing. 2010; 19(7-8): 998-1007. http://dx.doi.org/10.1111/j.136 5-2702.2009.02872.x

[18] Wong C, Lai KY, Tse HM. Effects of SP6 acupressure on pain and menstrual distress in young women with dysmenorrheal. ComplementaryTherapies in Clinical Practice. 2010; 16(2): 64-69. http: //dx.doi.org/10.1016/j.ctcp.2009.10.002
[19] GharloghiS, TorkzahraniS, AkbarzadehAR, et al. The effects of acupressure on severity of primary dysmenorrheal. Journal of Patient Preference and Adherence. 2012; (6): 137-142.

[20] Chang S, Jun EM, KangDH, et al. Effects of acupressure on dysmenorrhea and skin temperature changes in college students: a nonrandomized controlled trial. Int J Nurs Stud. 2007; 44(6): 973-81. http://dx.doi.org/10.1016/j.ijnurstu.2006.03.021

[21] Mirbagher AN, Adib HM, Mosaebi F. The effects of acupressure on primary dysmenorrhea: a randomized controlled trial. Complement Therapy Clinical Practice Journal. 2011; 17(1): 33-6. http://dx.doi.org/10.1016/j.ctcp.2010.06.005

[22] Kashefi F, Ziyadlou S, Khajehei M, et al. Effect of acupressure at the Sanyinjiao point on primary dysmenorrhea: a randomized controlled trial. Complement therapy clinical practice. 2010; 16(4): 198-202. http://dx.doi.org/10.1016/j.ctcp.2010.04.003 